\title{
The benefits of neuroplastic (self-directed) change versus abstinence for substance abusers: can neuroplastic changes be too late?
}

\section{Opinion}

There is an ideology that drug (including alcohol) abusers can with "therapy" induce a phenomenon widely known as self-mediated neuroplastic change. Such has been known to occur in patients suffering from permanent imbalance caused by certain medications who have healed completely. ${ }^{1}$ Would drug-induced neuroplastic change ever fail an addict, and why? Neuroplastic change occurs in the CNS and or brainstem to some degree, which can happen in the case of drug addiction. For certain individuals with a liver, for example, that has never reached the point of extensive fibrosis, there are many examples of alcoholic or users returning to moderation. ${ }^{1}$

The difficulty is that once a person has acute hepatitis, regardless of any neuroplastic change, any further intake of the substance will cause degradation of the tissues mentioned, hepatic or otherwise. ${ }^{2}$ While neuroplastic change, even self mediated, can induce significant change in behaviors, it cannot be recommended for all patients suffering from substance abuse as even moderate use will necessarily lead to degradation of hepatic or other tissues necessary for life without complete abstinence. Many physicians will instruct a substance abuser to never use that substance again, but fans of neuroplastic change explain that it may be possible to moderate their use. The reason physicians recommend abstinence is because once too much fibrosis, in the case of the liver, has set in one has obtained the condition "chronic hepatitis" (always swollen liver) accompanied by increased transaminases, usually AST and ALT is because that it is
Volume 3 Issue 4 - 2016

\author{
Amy J Austin \\ Freelance Tutor for Organic Chemistry, WyzAnt Tutoring, USA
}

Correspondence: Amy J Austin, Freelance Tutor for Organic Chemistry, WyzAnt Tutoring, USA,

Email amy.austin@DFTdeveloper.com

Received: April 20, 2016 | Published: April 26, 2016

risky to develop more fibrosis due to further consumption. ${ }^{2}$ This could happen with only one unit drink per day. ${ }^{3}$

My opinion is that if your physician has advised you to stop using any substance, to stop it immediately, to obtain counseling to support the emotional needs accompanying, and to follow your Physician's plan, regardless how difficult, as one does not want to ignore that for later dealing with the complications of cirrhosis or otherwise.

\section{Acknowledgements}

None.

\section{Conflict of interest}

The author declares no conflict of interest. 